\title{
强震前闭锁段周围应力场的某些特点
}

\author{
$丁$ 文 镜 \\ （国家地震局地质研究所）
}

1972 年召开的地应力会议发表了一篇探讨地震破坏模式的论文: “一个平面剪切破坏前 的应力场”. 该文认为地震孕育过程晚期, 断层填充物失去了抗剪能力. 地壳构造运动使断层 增长的剪切荷载都集中在闭锁段上. 由此可导出临震前地应力分布特征: 离震源稍远处总有 一个主应力指向震源(即闭锁段).

大量的强震观测资料表明, 浅源构造地震由于应力释放产生的 $P$ 波和 $S$ 波初动符号分布 图谱与双力偶源理论图谱一致 ${ }^{[1]}$. 因此，孕震期闭锁段积累应力的合力也应是双力偶. 文献 [1] 提出的模式是单力偶源. 如果计及断层厚度的作用, 就能把它变成双力偶源. 为此, 设断 层宽度为 $d$, 断层长度为 $2 L$, 断层闭锁长度为 $2 l$, 沿水平方向通过闭锁段切出薄平板 (图 1), 因其位于地壳深部, 上下表面都受压应力, 只能简化为平面应变状态. 如果断层面平均剪应力

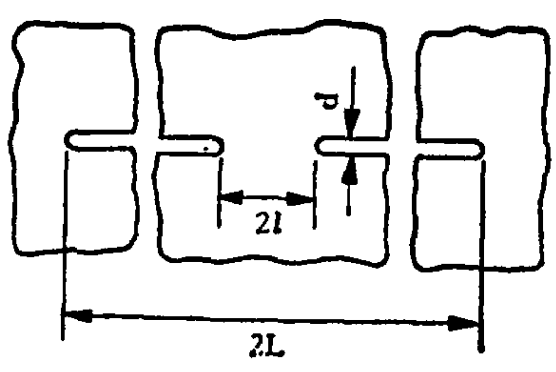

图 1

增量为 $q$, 平均压应力增量为 $p$. 通常, 闭锁段比断层短得 多, $l \ll L$. 所以,集中于闭锁段的剪切荷载增量可用集中 力 $Q=2 q L$ 代替. 按照圣维南原理,在离闭锁段足够远处 （大约 $1-2 l$ ), 分布荷载与集中荷载产生的应力无显著差 别. 关于压应力增量 $p$, 分别考虑两种特殊情况: 第一种 情况，压应力增量集中作用于闭锁段，这时，同样可用集中 力 $P=2 p L$ 代替压应力的作用. 第二种情况, 压应力增量 沿断层面均匀分布，相当于第一种情况迭加一个单向应力 场.

沿断层面将闭锁段中心部分切割出来 (图 2). 并且用集中力 $Q 、 P$ (或分布力 $p$ ) 和集中 力偶 $M$ 代替断层两盘对它的约束作用. 根据静力平衡原 理, $M=Q d$. 再用力的反作用原理, 就能作出两个半平面 的结构荷载图 (图 3). 由此可见, 只需分析半无限平板直 边上受集中力 $Q 、 P$ (或分布力 $p$ ) 和集中力偶 $M$ 的平面弹 性力学问题, 就能确定强震前闭锁段周围的应力分布.

选择集中力作用点为坐标原点 (图 4). 众所周知, 无 体积力的平面弹性力学问题归结为求解双调和方程 ${ }^{[2]}$

$$
\nabla^{2}\left(\nabla^{2} \phi\right)=0,
$$
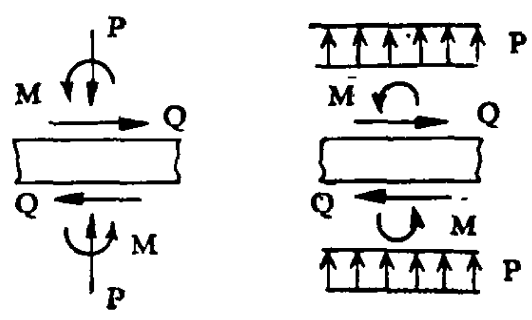

图 2

其中, 应力函数 $\phi$ 由边界条件决定. 第一种情况的应力函数 


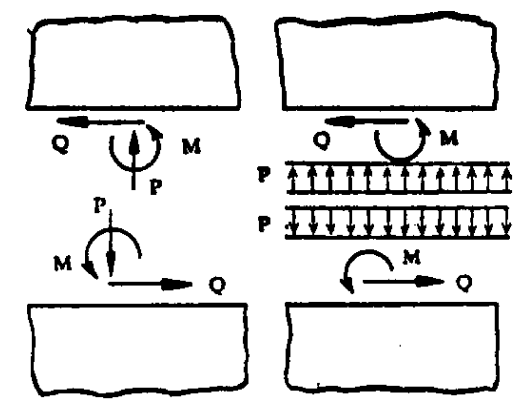

图 3
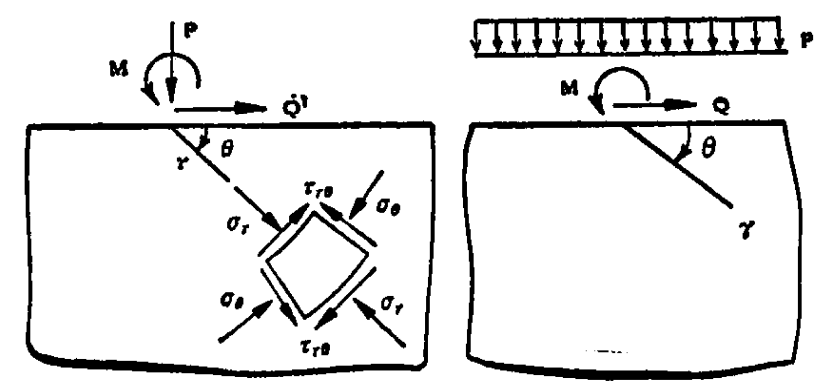

图 4

第二种情况的应力函数 $\phi=-\frac{Q}{\pi} r \theta \sin \theta+\frac{M}{\pi}[\theta-\cos \theta \sin \theta]+\frac{P}{\pi} r\left(\theta-\frac{\pi}{2}\right) \cos \theta$;

$$
\phi=-\frac{Q}{\pi} r \theta \sin \theta+\frac{M}{\pi}[\theta-\cos \theta \sin \theta]-\frac{p}{2} r^{2} \cos ^{2} \theta .
$$

将 $\phi$ 代入应力分量公式 $\sigma_{r}=\frac{1}{r} \frac{\partial \phi}{\partial r}+\frac{1}{r^{2}} \frac{\partial^{2} \phi}{\partial \theta^{2}}, \quad \sigma_{\theta}=\frac{\partial^{2} \phi}{\partial r^{2}}, \quad \tau_{r \theta}=-\frac{\partial}{\partial r}\left(\frac{1}{r} \frac{\partial \phi}{\partial \theta}\right)$,

求得应力分量的解析式. 第一种情况为

$$
\sigma_{r}=-\frac{4 q L}{\pi r} \cos \theta\left(1-\frac{d}{r} \sin \theta\right)-\frac{4 p L}{\pi r} \sin \theta, \sigma_{\theta}=0, \tau_{r \theta}=\frac{q d L}{\pi r^{2}}(1-\cos 2 \theta) ;
$$

第二种情况为

$$
\begin{aligned}
& \sigma_{r}=-\frac{4 q L}{\pi r} \cos \left(1-\frac{d}{r} \sin \theta\right)-p \sin ^{2} \theta, \\
& \sigma_{\theta}=-p \cos ^{2} \theta, \quad \tau_{r \theta}=\frac{q d L}{\pi r}(1-\cos \theta 2)-\frac{p}{2} \sin 2 \theta .
\end{aligned}
$$

根据实测的地应力数据, 利用以上结果可以确定断层和荷载参数. 首先, 设定断层半长 $L$ 和闭锁段位置. 决定测点位置参数 $r$ 和 $\theta$. 根据一个测点的应力值 $\sigma_{r} 、 \sigma_{\theta}$ 和 $\tau_{r \theta}$, 用 (6) 式可 算出荷载 $p$ 和 $q$, 以及断层宽度 $d$. 然后, 利用几个测点的 $\sigma_{r}, \sigma_{\theta}$ 和 $\tau_{r \theta}$ 值, 根据误差取极小的 原则, 调整原先设定的断层半长和闭锁位置. 最终可以得到合理的结果.

为了作定性分析, 引人下列参数

$$
\bar{r}=\frac{r}{d}, \quad \alpha=\frac{\pi d}{L}, \quad \beta=\frac{p}{q}, \quad \tau_{0}=\frac{q}{\alpha},
$$

将 (5) 式和 (6) 式分别表示为

$$
\begin{aligned}
& \sigma_{r}=-4 \tau_{0} \cos \theta(\bar{r}-\sin \theta) \bar{r}^{-2}-4 \beta \tau_{0} \sin \theta \bar{r}^{-1}, \\
& \sigma_{\theta}=0, \tau_{r \theta}=\tau_{0}(1-\cos 2 \theta) \bar{r}^{-2}
\end{aligned}
$$

和

$$
\begin{aligned}
& \sigma_{r}=-4 \tau_{0} \cos \theta(\bar{r}-\sin \theta) \bar{r}^{-2}-\alpha \beta \tau_{1} \sin ^{2} \theta, \\
& \sigma_{\theta}=-\alpha \beta \tau_{0} \cos ^{2} \theta, \tau_{r \theta}=\tau_{0}(1-\cos 2 \theta) \bar{r}^{-2}-\frac{1}{2} \alpha \beta \tau_{0} \sin 2 \theta .
\end{aligned}
$$

通常, 断层宽度为几十米, 比断层长度小得多, 而且, 发震断层走向与区域应力场主方向间 夹角偏离 $45^{\circ}$ 不会太远. 因此, 取下列典型数据 $\alpha=0.01 ; \beta=0.5 、 1 、 2$ 做数值计算, 最后将 主应力迹线分别表示于图 5 和图 6. 其中, 虚线是最大主应力(张性应力)迹线. 实线是最小 主应力(压性应力)迹线。 

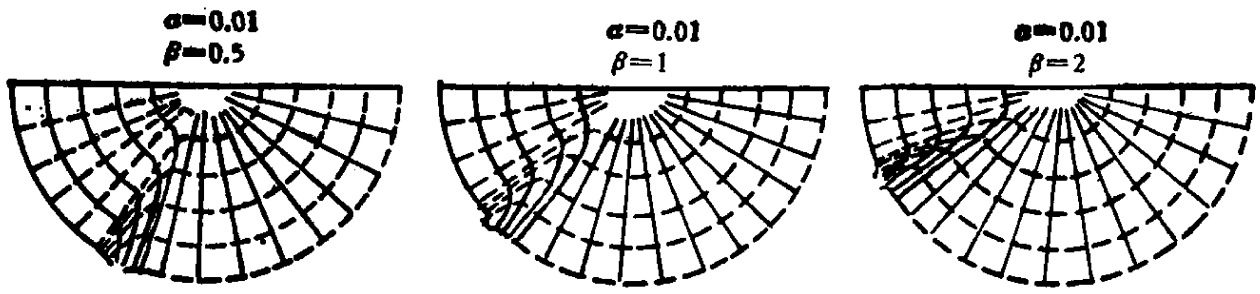

图5
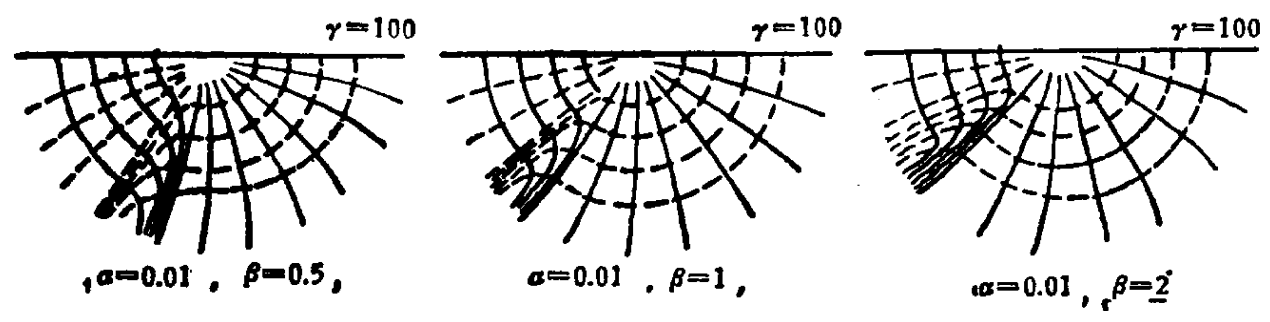

图 6

对比图 5 和图 6 中的曲线,在许多地区，上述两种特殊情况的主应力迹线存在显著差别. 第一种情况（图 5)，一切离闭锁段足够远的地点, 例如, $\bar{r}>10$, 半平面上除去

$$
\theta=\operatorname{tg}^{-1}\left(\frac{-1}{\beta}\right)
$$

附近很狭窄的扇形区外，总有一个主应力指向闭锁段. 第二种情况（图 6)，在 $100>\bar{r}>10$ 的环形带上，除去 $\theta=\operatorname{tg}^{-1}\left(\frac{-1}{\beta}\right)$ 附近的一个扇形区域外，同样有一个主应力指向闭锁段. 但是，离开闭锁段更远的点，由于分布压应力 $p$ 的作用,随着 $\bar{r}$ 增加，保持主应力指向闭锁段的 区域越来越小.显然, 当 $\vec{r} \gg 100$ 时,除去 $\theta=0^{\circ}$ 和 $\theta=180^{\circ}$ 附近较小扇形区外, 主应力将不 再指向闭锁段了. 在地壳中, 由于断层闭锁䝘岩体比较完整, 刚度较大, 闭锁段上总要产生压应 力集中现象. 所以,压应力沿断层分布的真实状况应介于上述两种特殊情况之间, 从而扩大了 主应力指向闭锁段区域的范围. 实践表明, 许多次大地震震前发现地应力主方向汇交于震源. 例如, 1973 年的炉霍地震, 1974 年的大关地震, 1975 年的海城地震和 1976 年的唐山地震 ${ }^{[2,3]}$. 按照以上分析结果, 说明这些地震的震源存在强大的闭锁段.

为了研究远离闭锁段地点主应力数值变化的规律. 在 (8) 式中略去 $\bar{r}^{-2}$ 项, 仅保留 $\bar{r}^{-1}$ 项. 从而得到近似公式

$$
\sigma_{r} \approx-4 \tau_{0}(\cos \theta+\beta \sin \theta) \bar{r}^{-1}, \quad \sigma_{\theta}=0, \quad \tau_{r \theta} \approx 0 .
$$

上式表明, $\sigma_{\theta} \approx \sigma_{1}=0, \sigma_{r} \approx \sigma_{2}<0$, 并且, $\sigma_{r}$ 与 $\bar{r}$ 成反比. 由方程式 $\frac{\partial \sigma_{r}}{\partial \theta}=0$,

可以求得 $\theta$ 为常值. $\sigma_{r}$ 取极值地点的坐标角 $\theta_{1}=\operatorname{tg}^{-1} \beta$, 同时, 令 $\sigma_{r}=0$, 可以求得 $\sigma_{r}$ 取零值 地点的角坐标

$$
\theta_{2}=\operatorname{tg}^{-1}\left(-\frac{1}{\beta}\right)
$$

不难证明, $\theta_{1}$ 和 $\theta_{2}$ 间有关系式 $\theta_{2}=\theta_{1}+\frac{\pi}{2} \quad(\beta>0), \theta_{2}=\theta_{1}-\frac{\pi}{2} \quad(\beta<0)$.

利用以上结果, 可以定性地描绘出 $\sigma_{r}$ 的等值线图 (图 7). 该图可粗略划分地应力变化的 
灵敏区和不灵敏区.

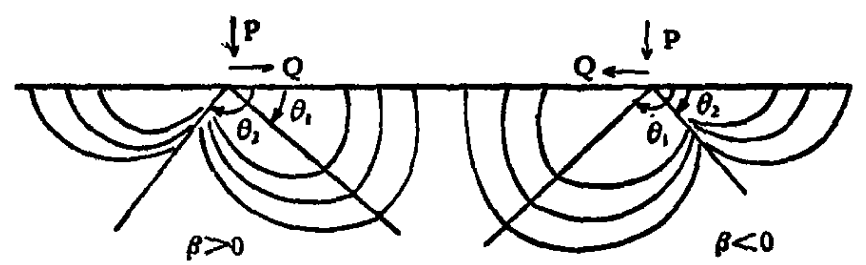

图 7

反过来, 根据实测的地应力 (相对值) 分布状况, 利用图 7 中曲线, 可以推测出荷载应力增 量 $\boldsymbol{p}$ 和 $q$ 的相对大小. 从而粗略估计出发震前地壳中新增压缩荷载的方向.

综合以上分析,只需在闭锁段周围, 在地应力变化比较灵敏 (参照图 7), 而且主应力方向 可能指向闭锁段的地区(参照图 5 和图 6), 设置若干地应力测点, 同时测量这些地点的地应力 (相对值),计算出相应的主方向. 只要发现主应力方向明显地汇交, 就表明汇交点附近形成了 闭锁段,可能成为未来强震的震中区. 据此就可提出地震预报的意见.

上述分析将岩石作为理想弹性体, 地壳深部岩石处于高温高压状态, 可能具有粘弹介质特 性. 但是,按照钱学森推广 Alfrey 对应定理得到的结论：线性粘弹介质中的应力分布和纯弹 性介质在同一瞬时的表面力和体积力作用下的应力分布相同 ${ }^{[3]}$. 因此，上述结论对地壳深部 岩石仍应成立。

谢致：承蒙王仁和张文佑教授对本文的审阅和指正，作者表示诚揧的感谢.

\title{
）文 献
}

[1] Khattri, K., Earth-Science Rev., 9(1973), 19-63.

[2] Timoshenko, S. \& Goodier, J. N., Theory of Elastioity, 1951.

[3] Tsien, H. S. (钱学森), Quart. Appl. Math., 8(1950), 104-106.

\section{SOME CHARACTERISTICS OF STRESS FIELD AROUND BLOCK. SEGMENTS IMMEDIATELY BEFORE STRONG SHOCK WAVES TAKE PLACE}

\author{
DiNG WEN-JING (丁文镜)
}

\begin{abstract}
This paper deals with some characteristics of the stress field around the block-segments of faults immediately before strong shock waves take place. Analytic results show that the main additional stresses are all directed at the block-segments of faults with the exception of some narrow areas. This has been confirmed by data on the stresses measured a number of times immediately before some strong shocks. Thus, dangerous zones of strong earthquakes can be determined if precise measurements of ground stresses are available.
\end{abstract}

